\title{
Training schedule in a two-turn task in a temporal circular maze
}

\author{
CHIA-SHONG CHEN 1 \\ THE AUSTRALIAN NATIONAL UNIVERSITY, CANBERRA
}

Effectiveness of three training schedules on learning of a two-turn task in a temporal circular maze was studied with white rats. The results were viewed as giving support to a working hypothesis that the degree of learning was the result of the interaction between guided and free trials.

It was suggested in a previous study (Chen, 1967) that a temporal circular maze which consisted of a circular runway and a radial stem might provide a useful technique for systematic studies of the teaching or guidance process in learning, an important field which has not been extensively studied by comparative psychologists (see Holding, 1965)。In that study, S was required, after emerging from the stem, to follow the runway for two turns in one direction before reentering the stem for food (two turn task). The results of that study showed that for white rats, a certain degree of guidance was required if learning was to occur. A trial and error method was quite ineffective. Furthermore, it was shown that a training schedule with Guided/Free trial (G/F) ratio of one was more effective in training white rats than schedules for which this ratio was smaller than one.

The purpose of the present experiment was to compare, for the same task, the effectiveness of a training schedule with a $G / F$ ratio of two with two different schedules where the $G / F$ ratio is one. Thus a $3 x$ $4 \mathrm{G} 2 \mathrm{~F}(\mathrm{G} / \mathrm{F}=2)$ (i.e., three repetitions of a block of four guided trials followed by two free trials), a $3 \mathbf{x}$ $4 G 4 F(G / F=1)$, and a $3 \times 2 G 2 F(G / F=1)$ schedule were employed. The effectiveness of the training schedules was assessed in two ways: (1) relative rate of reaching a predetermined criterion of learning, and (2) the efficiency of the postcriterion performance. Subjects

The Ss were 24 male white rats about 18 weeks old at the beginning of the experimental training. They were housed in groups of eight with water freely available, and were given an average of $8 \mathrm{~g}$ per $\mathrm{S}$ (64 $\mathrm{g}$ per group) of dry rat food soon after all Ss in each group had completed their daily training.

\section{Apparatus}

Figure 1 shows the ground plan of the maze. D represents a sliding door which was used as a mechanical means of guiding S's response; FT is a food tray into which the reward (1 cm of raw spaghetti) was delivered from a Deutsch Spaghetti Gun (SG). The letter S shows the approximate spot where $S$ was put into the stem at the beginning of each training session. The stem served as a start box at the beginning of a trial and as an end box at the end of the trial. A trial was said to have occurred when S ran out of the stem, followed the runway for at least one complete turn, and reentered the stem; a trial ended only when $S$ interrupted the beam to Photocell 3.

\section{Procedure}

On guided trials the door $\mathrm{D}$ was closed as soon as $S$ entered the runway and reopened when it was about to complete the second turn; thus $S$ was forced to make a correct trial. On free trials the door was kept open all the time and there was no extraneous interference, so that $\mathbf{S}$ was free to make either a correct or an erroneous trial. Each daily session began by putting

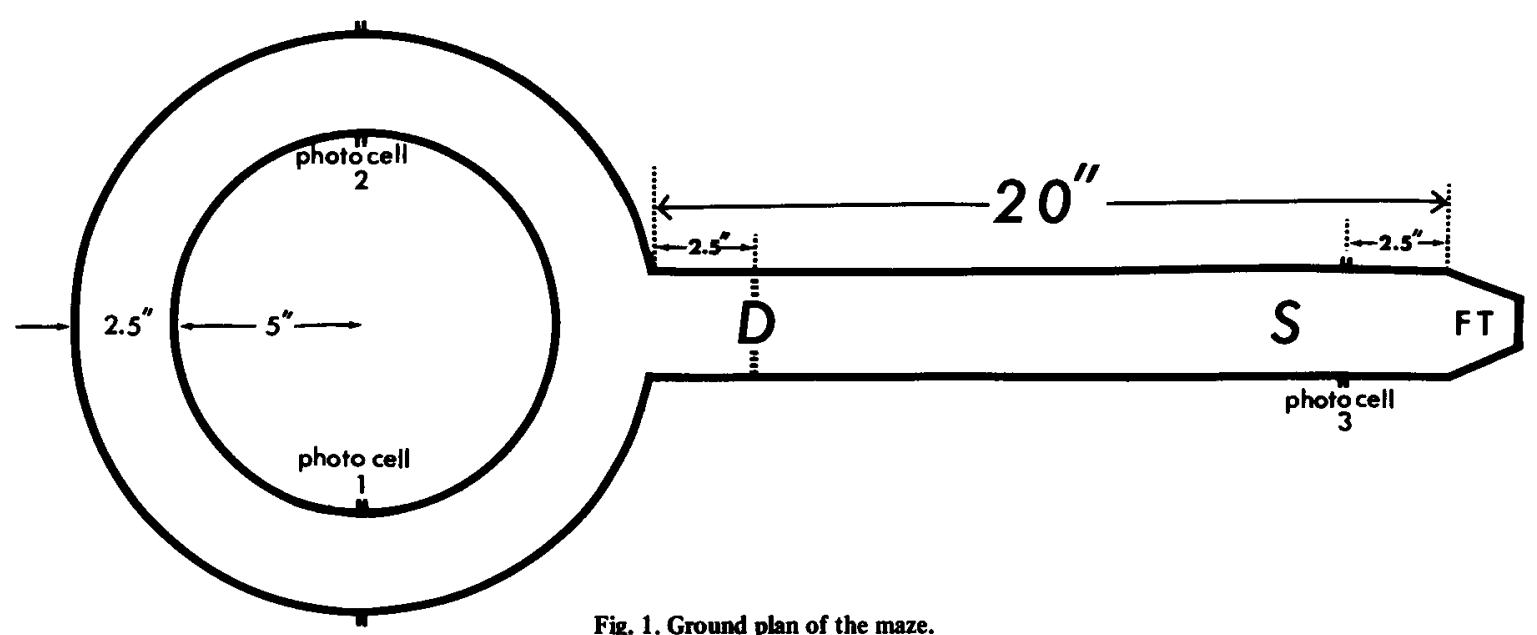


$S$ into the stem and ended when the assigned number of daily trials were finished. On correct and guided trials spaghetti was delivered as soon as $S$ interrupted the beam to Photocell 3 ; no punishment was administered at the end of erroneous trials.

In preliminary training, $S$ was first trained in a $4 \times 4 \times 19$ in. feeding box for eight days to habituate it to the noise of the operation of a SG. On each day $S$ was allowed to stay in the box until five pieces of spaghetti were consumed. After feeding training, all Ss were transferred to the temporal maze and were run five trials a day for nine days to familiarize them with the maze. Spaghetti was given from SG at the end of each trial irrespective of S's performance.

Experimental training. After preliminary training, Ss were randomly divided into three equal groups, and were trained under the $3 \times 4 G 2 F$ (Group I), $3 \times$ 4 G 4 F (Group II), and $3 \times 2$ G 2 F (Group III) schedules. However, during the course of training minor variations from the schedules were made. In particular: (1) When the last free trial of each block was a correct one, an extra free trial (or trials, as the case might be) was given. (2) If $\mathrm{S}$ made two or more (but less than five) consecutive correct runs, further free trials were run until two consecutive errors occurred. The criterion of learning was five consecutive correct runs; guidance was discontinued when the criterion was reached. On the criterion session, 20 extra free trials were given immediately following the fifth criterion trial. On following sessions, each $S$ was run in a daily session of 30 free trials, and sessions were continued until a certain level of stable performance was reached. Stability of performance was said to have been attained: (1) when $S$ made at least $80 \%$ correct (i.e., at least 24 correct trials) on each session for five consecutive sessions, and (2) when the range of the number of correct trials on these five sessions was not larger than 4. These criteria of stability were determined before the experiment started.

\section{Results and Discussion}

Acquisition. The overall comparison of groups was tested, throughout the following section, by the KruskalWallis one way analysis of variance by ranks. The medians of the total number of trials required to reach the criterion of learning were 290 (range 206642), 341 (range 327-497), and 327.5 (range 242-441) for Groups I, II, and III, respectively. Statistical analysis indicated no significant effect over the groups $(\mathrm{H}=2.34, \mathrm{df}=2, \mathrm{p}>.30$ ). However, a Bartlett test indicated significant heterogeneity of variance among groups $(B=7.16, d f=2, p<.05)$. $F$ tests indicated that the variance of Group I was significantly larger than that of Group II $(F=5.84, d f=7 / 7, p<.05)$ or Group III $(F=6.22$, df $=7 / 7, p<.05$ ), and there was no significant difference in the variance of Groups II and III, which were trained under the schedules with $G / F=1(F=1.07$, $\mathrm{df}=7 / 7, \mathrm{p}>.10)$.

Postcriterion performance. The three groups were compared in terms of the number of correct trials made by each $S$ in the first 50 postcriterion trials. The medians for this score were 38.5 (range 33-44), 39.5 (range 34-47), and 33 (range 28-36) for Groups I, II, and III, respectively; the effect over the groups was significant $(H=10.12, d f=2, p<.01)$. Mann-Whitney $U$ tests showed that Groups I and II did not differ significantly from each other, but they were significantly better than Group III $(U=7, p=.006$ and $U=6, p=.004)$.

The medians of the number of postcriterion sessions required to the stability criteria were 0 (range $0-8$ ), 1.5 (range $0-10$ ), and 3 (range $1-7$ ) for Groups I, II, and III, respectively; the overall effect was not significant ( $\mathrm{H}=5.64, \mathrm{df}=2, \mathrm{p}<.10)$. Although the analysis of variance failed to reach significance, it is worth noting that Group I was significantly better than Group III (U $=9, p=.014)$.

The medians of the range of the number of correct trials made on the first 10 postcriterion sessions were 4 (range $4-12$ ), 7 (range 5-14), and 8.5 (range 7-23) for Groups I, II, and III, respectively; the overall effect was significant $(\mathrm{H}=8.98, \mathrm{df}=2, \mathrm{p}<.02)$. MannWhitney $U$ tests showed that Group I was significantly better than Groups II and III ( $U=12, p=.038$ and $U$ $=8, p=.01$ ). This result seems to suggest that the performance of Ss trained under schedules with $G / F$ $=1$ was less stable than those trained under the schedule with $G / F=2$.

The above results were viewed as giving some support to a working hypothesis that the effectiveness of training schedules cannot be accounted for entirely in terms of the number of guided or free trials alone, but rather it is the interaction of the two which is important. If the number of guided trials alone were important, then no differences would be expected between Groups I and II which received equal numbers of guided trials each day. On the other hand, if the free trials alone were essential, then there should be no differences between Groups I and III, which received equal numbers of free trials each day. It appears that the temporal circular maze used may provide a useful tool for objective study of some important aspects of behavioral shaping.

\section{References}

CHEN, C. S. Guidance in a two-turn task in a temporal circular maze. J. comp. physiol. Psychol, 1967, in press.

HOLDING, D. H. Principles of training. New York: Pergamon Press, 1965. Pp. 39-42. Note

1. A. N. U. Postgraduate research scholar. I thank Dr. J. R. Trotter for discussions, and Professor C. A. Gibb for his encouragement and for the facilities of his department. 